\title{
The human brain in numbers: a linearly scaled-up primate brain
}

\section{Suzana Herculano-Houzel*}

Instituto de Ciências Biomédicas, Universidade Federal do Rio de Janeiro, Rio de Janeiro, Brasil

\section{Edited by:}

Andreas Jeromin

Allen Institute for Brain Science, USA

Reviewed by:

Karl Herrup

Case Western University, USA

Robert Barton,

University of Durham, UK

\section{${ }^{*}$ Correspondence:}

Suzana Herculano-Houzel, Laboratório

de Neuroanatomia Comparada,

Instituto de Ciências Biomédicas,

Universidade Federal do Rio de

Janeiro, Rua Carlos Chagas Filho 373,

21950-902 Rio de Janeiro,

Rio de Janeiro, Brazil.

e-mail:suzanahh@gmail.com
The human brain has often been viewed as outstanding among mammalian brains: the most cognitively able, the largest-than-expected from body size, endowed with an overdeveloped cerebral cortex that represents over $80 \%$ of brain mass, and purportedly containing 100 billion neurons and $10 \times$ more glial cells. Such uniqueness was seemingly necessary to justify the superior cognitive abilities of humans over larger-brained mammals such as elephants and whales. However, our recent studies using a novel method to determine the cellular composition of the brain of humans and other primates as well as of rodents and insectivores show that, since different cellular scaling rules apply to the brains within these orders, brain size can no longer be considered a proxy for the number of neurons in the brain. These studies also showed that the human brain is not exceptional in its cellular composition, as it was found to contain as many neuronal and non-neuronal cells as would be expected of a primate brain of its size. Additionally, the so-called overdeveloped human cerebral cortex holds only $19 \%$ of all brain neurons, a fraction that is similar to that found in other mammals. In what regards absolute numbers of neurons, however, the human brain does have two advantages compared to other mammalian brains: compared to rodents, and probably to whales and elephants as well, it is built according to the very economical, space-saving scaling rules that apply to other primates; and, among economically built primate brains, it is the largest, hence containing the most neurons. These findings argue in favor of a view of cognitive abilities that is centered on absolute numbers of neurons, rather than on body size or encephalization, and call for a re-examination of several concepts related to the exceptionality of the human brain.

Keywords: brain scaling, number of neurons, human, encephalization

\section{INTRODUCTION}

\section{THE HUMAN BRAIN AS A SPECIAL BRAIN}

What makes us human? Is our brain, the only one known to study other brains, special in any way? According to a recent popular account of what makes us unique, "we have brains that are bigger than expected for an ape, we have a neocortex that is three times bigger than predicted for our body size, we have some areas of the neocortex and the cerebellum that are larger than expected, we have more white matter" - and the list goes on (Gazzaniga, 2008). Most specialists seem to agree (for example, Marino, 1998; Rilling, 2006; Sherwood et al., 2006). Since ours is obviously not the largest brain on Earth, our superior cognitive abilities cannot be accounted for by something as simple as brain size, the most readily measurable parameter regarding the brain. Emphasis is thus placed on an exceptionality that is, curiously, not brain-centered, but rather body-centered: With a smaller body but a larger brain than great apes, the human species deviates from the relationship between body and brain size that applies to other primates, great apes included, boasting a brain that is $5-7 \times$ too large for its body size (Jerison, 1973; Marino, 1998). Recent efforts to support this uniqueness have focused on finding genetic differences between humans and other primates (reviewed in Vallender, 2008), as well as cellular particularities such as the presence and distribution of Von Economo neurons (Nimchinsky et al., 1999; but see Butti et al., 2009; Hakeem et al., 2009).

To regard the human brain as unique requires considering it to be an outlier: an exception to the rule, whatever that rule is. This makes little sense, however, in light of evolution. If we go to such great lengths to affirm, and teach, that evolution is the origin of diversity in life, and to find trends and laws that apply to kingdoms, phyla and orders as a whole, why then insist that whatever scaling rules apply to other primates must not apply to us? In view of the vexing size inferiority in brain size and of the lack of information about what our brains are actually made of - and how that compares to other brains, particularly those of whales and elephants - resorting to a quest for uniqueness may have seemed as a necessary, natural step to justify the cognitive superiority of the human brain.

Recently, a novel quantitative tool developed in our lab (Herculano-Houzel and Lent, 2005) has finally made the numbers of neurons and non-neuronal cells that compose the brains of various mammals, humans included, available for comparative analysis. This review will focus on such a quantitative, comparative analysis, with emphasis on the numbers that characterize the human brain: what they are, how they have been viewed in the past, and how they change our view of where the human brain fits into the diversity of the mammalian nervous system.

\section{THE HUMAN BRAIN IN NUMBERS}

How many neurons does the human brain have, and how does that compare to other species? Many original articles, reviews and textbooks affirm that we have 100 billion neurons and 10 times more glial cells (Kandel et al., 2000; Ullian et al., 2001; Doetsch, 2003; Nishiyama et al., 2005; Noctor et al., 2007; Allen and Barres, 2009), usually with no references cited. This leaves the reader with 
the impression that the cellular composition of the human brain has long been determined. Indeed, an informal survey with senior neuroscientists that we ran in 2007 showed that most believed that the number of cells in the human brain was indeed already known: that we have about 100 billion neurons, outnumbered by about 10 times more glial cells - but none of the consulted scientists could cite an original reference for these numbers (Herculano-Houzel and Lent, unpublished observations). Curiously, the widespread concept that neurons represent about $10 \%$ of all cells in the human brain might be one of the arguments behind the popular, but mistaken, notion that we only use $10 \%$ of our brain (Herculano-Houzel, 2002).

The reason for such lack of references is that indeed there was, to our knowledge, no actual, direct estimate of numbers of cells or of neurons in the entire human brain to be cited until 2009. A reasonable approximation was provided by Williams and Herrup (1988), from the compilation of partial numbers in the literature. These authors estimated the number of neurons in the human brain at about 85 billion: $12-15$ billion in the telencephalon (Shariff, 1953), 70 billion in the cerebellum, as granule cells (based on Lange, 1975), and fewer than 1 billion in the brainstem. With more recent estimates of 21-26 billion neurons in the cerebral cortex (Pelvig et al., 2008) and 101 billion neurons in the cerebellum (Andersen et al., 1992), however, the total number of neurons in the human brain would increase to over 120 billion neurons.

As to the 10 times more numerous glial cells in the human brain, that seems to be the case only in subcortical nuclei such as the thalamus ( 17 glial cells per neuron) and the ventral pallidum (12 glial cells per neuron; Pakkenberg and Gundersen, 1988). In the gray matter of the cerebral cortex, glial cells outnumber neurons by a factor of $<2$ (Sherwood et al., 2006; Pelvig et al., 2008). Given the relatively small number of glial cells reported for the human cerebellum, where they are outnumbered by neurons by at least 25:1 (Andersen et al., 1992), the only possible explanation for the ubiquitous quote of 10 times more glial than neuronal cells in the entire human brain would be the presence of nearly one trillion glial cells in the remaining structures alone - an unlikely scenario, since these structures represent $<10 \%$ of total brain mass.

\section{WHY BOTHER WITH CELL NUMBERS?}

Across species, the number of neurons and their relative abundance in different parts of the brain is widely considered to be a determinant of neural function and, consequently, of behavior (Williams and Herrup, 1988). Among mammals, those species with the largest brains, such as cetaceans and primates, have a greater range and versatility of behavior than those with the smallest brains, such as insectivores (Jerison, 1985; Marino, 2002). Among birds, those that are larger-brained (corvids, parrots and owls) are also considered the most intelligent (Lefebvre et al., 2004). A recent comparison of several parameters, including brain size, relative brain size, encephalization, conduction velocity and estimated numbers of neurons led two authors to conclude that the "factors that correlate better with intelligence (across species) are the number of cortical neurons and conduction velocity, as the basis for information processing" (Roth and Dicke, 2005). Indeed, within non-human primates, a recent meta-analysis concluded that the best predictor of the cognitive abilities of a species is absolute brain size, not relative size nor encephalization quotient (EQ; Deaner et al., 2007).
However, the correlation between absolute brain size and cognitive abilities breaks down when species of similar brain size are compared across orders. Monkeys, for instance, possess brains that are much smaller than those of ungulates, but the higher cognitive and behavioral flexibility of monkeys over ungulates is anecdotally evident to any observer who compares the ingenious and complex abilities of macaques to those of cows or horses, even though the latter have $4-5 \times$ larger brains than macaques. For similar-sized brains, rodents also perform more poorly than primates: With a brain of only $52 \mathrm{~g}$, the behavioral, social and cognitive repertoire of the capuchin monkey is outstanding compared to the capybara, a giant Amazonian rodent (MacDonald, 1981), even though the latter has a larger brain of $75 \mathrm{~g}$. This is reminiscent of the most striking and troubling discrepancy regarding brain size and cognitive abilities: that between humans and larger-brained species such as whales and elephants. If the latter have brains that are up to six times larger than a human brain, why should we be more cognitively able? Answering this question requires a direct examination of the numbers of neurons that compose the brains of humans and other species.

\section{BRAIN AND BODY SCALING: THE TRADITIONAL VIEW ASSUMPTION 1: BODY SIZE MATTERS}

If the smaller size of the human brain compared to elephant and whale brains (Figure 1) translates into a smaller number of neurons in the human brain than in the latter, then what makes the human brain outstanding in its cognitive abilities? In the absence of direct estimates of numbers of neurons in these and other species, the search for a neural correlate for human capacities has placed emphasis on the characteristic that most undisputedly places humans above other mammals: the EQ (Jerison, 1973). This measure is based on the observation that, across species, brain size correlates with body size in a way that can be described mathematically with a power function, thus allowing the predicted brain mass to be calculated for any species. EQ indicates how much the observed brain mass of a species deviates from the expected for its body mass: an EQ of 1 indicates that the observed brain mass matches the expected value; an EQ $>1$ means that brain size in that species is larger than expected for its body mass.

Compared to mammals as a whole, humans have the largest EQ found for any mammal, of between 7 and 8 (Jerison, 1973); even if compared to anthropoid primates only, humans still have an EQ of over 3, a value that is larger than that obtained for any other
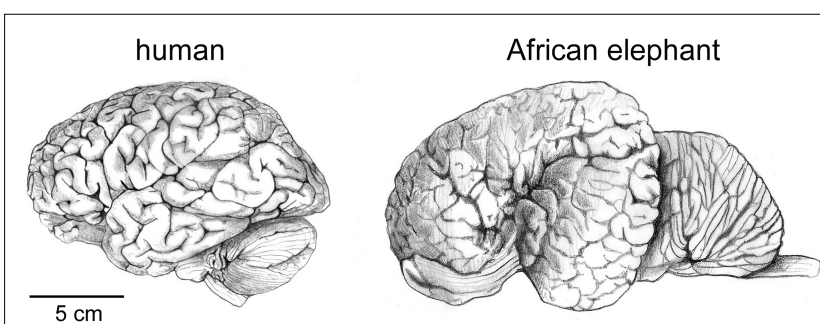

FIGURE 1 |The human brain is not the largest. Brains of a human and of an African elephant are depicted here at the same scale. Drawings by Lorena Kaz based on images freely available from the University of Wisconsin and Michigan State Comparative Mammalian Brain Collections (www.brainmuseum.org). 
primate or cetacean (Marino, 1998). The position of the human species as an outlier in the body $\times$ brain comparison is made clear if one considers that although gorillas and orangutans overlap or exceed humans in body size, their brains amount to only about one-third of the size of the human brain.

There are, however, several problems with the notion that the explanation for the superior cognitive abilities of the human species lies in its large EQ. For one, it is not obvious how largerthan-expected brain mass would confer a cognitive advantage. In principle, this advantage would rely on the availability for cognitive functions of whatever brain mass exceeds what is necessary to process body-related information. However, according to this notion, small-brained animals with very large EQs should be expected to have more cognitive abilities than large-brained animals with smaller EQs. Capuchin monkeys, for instance, have much larger EQs than gorillas (Marino, 1998), but are outranked by these in cognitive performance (Deaner et al., 2007). Absolute brain mass and number of neurons, left out of the encephalization equation, must clearly be taken into consideration, since the "exceeding number of neurons" in a large brain should necessarily be larger than that in a smaller brain of same EQ (Herculano-Houzel, 2007).

Another problem with the utility of the EQ is that the bodybrain mass relationship from which expected brain mass is derived depends on the precise combination of species computed (Barton, 2006; Herculano-Houzel et al., 2007). We have recently found that, compared to the linear brain $\times$ body relationship that applies to the primate species in our sample (which consisted of simian and prosimian primates; Herculano-Houzel et al., 2007), the human brain deviates by only $10 \%$ from its expected size (Azevedo et al., 2009). This conformity to the body $\times$ brain relationship that applies to non-anthropoid primates is consistent with the observation that, like in other non-anthropoid primates, the human brain mass represents about $2 \%$ of body mass. Given the sensitivity of EQ to the species included and our finding that the human brain conforms to the scaling rules that apply to other primates (see below), we have suggested that, rather than humans having a larger brain than expected, it is the great apes such as orangutans and, more notably, gorillas that have bodies that are much larger than expected for primates of their brain size (Herculano-Houzel et al., 2007).

This latter possibility of a dissociation between brain and body development in evolution (which might be only circumstantially, and not causally, related) constitutes a final criticism to the usefulness of the EQ as an index of "brain" evolution in comparative studies: indeed, the emphasis on the body-centered EQ overlooks the observation that, compared to other mammalian orders, primate encephalization is the result of a shift in postcranial growth processes, not a modification of brain growth (Deacon, 1997). In the words of Deacon, "if primates have big brains merely because they have small bodies, we cannot presume that this represents an evolutionary trend driven by cognitive demands"(p. 343). In this scenario, however, the human brain exhibits a further modification in that it continues to grow as though in a larger body (Deacon, 1997).

\section{ASSUMPTION 2: BRAIN SIZE MATTERS}

Brain size varies across mammals by a factor of approximately 100,000 (Tower, 1954; Stolzenburg et al., 1989). Different mammalian orders have traditionally been pooled together in studies of brain allometry, as if their brains were built according to the same scaling rules (for example, Haug, 1987; Zhang and Sejnowski, 2000).

Comparisons across orders that seem to invalidate the correlation between numbers of neurons and cognitive ability, such as between monkeys and ungulates, or rodents and primates, also bear this hidden caveat: the assumption that brain size relates to number of neurons in the brain in a similar fashion across orders. This assumption, which was justifiable by the lack of direct estimates of the neuronal composition of the brain of different species, is so widespread that it implicitly or explicitly underlies most comparative studies to date (for example, Haug, 1987; Finlay and Darlington, 1995; Barton and Harvey, 2000; Clark et al., 2001). The very concept of encephalization presupposes that not only the brain scales as a function of body size, but that all brains scale the same way, such that the only informative (and sufficient) variable is brain size and its deviation from the expected. However, our quantitative studies on the cellular scaling rules that apply to different mammalian orders have shown that this assumption is invalid and therefore should no longer be applied (see below).

\section{ASSUMPTION 3: PROPORTIONS AND RELATIVE SIZE MATTER}

An often cited argument in favor of the uniqueness of the human brain is its relatively large cerebral cortex, which accounts for $82 \%$ of brain mass. Within this large cerebral cortex, a relative enlargement of the prefrontal cortex was once considered a hallmark of the human brain, but this view has however been overthrown by modern measurements (Semendeferi et al., 2002). Still, the distribution of cortical mass in humans may differ from that in other primates, endowing particularly relevant regions such as area 10 with relatively more neurons in the human cortex (Semendeferi et al., 2001).

Relative size is supposed to be a meaningful indicator of relative functional importance of a brain structure based on the assumption that it is a proxy for relative number of neurons. For instance, the increase in relative size of the cerebral cortex with increasing brain size simultaneously with no systematic change in the relative size of the cerebellum has been used as evidence that these structures are functionally independent and have been evolving separately (Clark et al., 2001). Such discrepancy would support the popular notion that brain evolution equates with development of the cerebral cortex, which comes to predominate over the other brain structures. However, analysis of absolute, rather than relative, cerebral cortical and cerebellar volumes in the same dataset leads to the opposite conclusion: the coordinated scaling of these volumes, as well as of the surface areas of these structures, would be evidence that the cerebral cortex and cerebellum are functionally related and have been evolving coordinately (Barton, 2002; Sultan, 2002).

As it turns out, however, the underlying assumption that the relative size of a brain structure reflects the relative number of brain neurons that it contains is flawed.

Now that numbers of neurons are available across rodents, primates and insectivores, we find that the cerebral cortex, despite varying in relative size from $42 \%$ (in the mouse) to $82 \%$ of brain mass (in the human), contains between 13 and $28 \%$ of all brain neurons in 15 of 18 species studied, ranging between 13\% (in moles) and $41 \%$ (in the squirrel monkey; Herculano-Houzel et al., 
2006, 2007; Sarko et al., 2009). Most importantly, this fractional number of neurons in the cerebral cortex relative to the whole brain is not correlated with the relative size of the cerebral cortex (Figure 2). Instead, the number of neurons in the cerebral cortex increases coordinately with the number of neurons in the cerebellum (Herculano-Houzel, submitted).

\section{A NEW VIEW: SCALING OF NEURONAL NUMBERS CELLULAR SCALING RULES FOR RODENT, INSECTIVORE, AND PRIMATE BRAINS}

Our group has been investigating the cellular scaling rules that apply to brain allometry in different mammalian orders using the novel method of isotropic fractionation, which produces cell counts derived from tissue homogenates from anatomically defined brain regions (Herculano-Houzel and Lent, 2005). Through the estimation of absolute numbers of neuronal and non-neuronal cells in the brains of different mammalian species and their comparison within individual orders, we have been able to determine the scaling rules that apply to the brains of species spanning a wide range of body and brain masses in rodents (Herculano-Houzel et al., 2006), primates (Herculano-Houzel et al., 2007) and more recently in insectivores (Sarko et al., 2009). A comparative overview of brain mass and total number of neurons for these species can be seen in Figure 3.

A recent issue in comparative studies of brain scaling has been the examination of how residual variation in different parameters relate to phylogenetic relationships once shared evolutionary commonalities in body or brain size are accounted for (Harvey and Pagel, 1991; Nunn and Barton, 2000). Although such analyses of independent contrasts are instrumental for identifying evolutionary correlations across taxa while taking into account this phylogenetic nonindependence, they overlook the very issue at hand here: how the size of the brain reflects the number of neurons that it contains, regardless of body size and of any other shared characteristics. For this reason, the analysis reviewed here, referred to as unveiling the "cellular scaling rules" for the brain of different mammalian

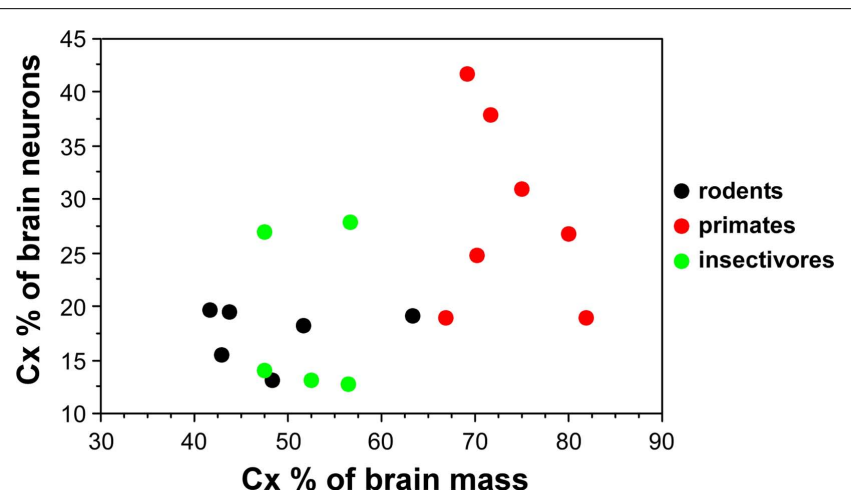

FIGURE 2 | Relative size of the cerebral cortex does not inform about the relative number of neurons in the cortex compared to the whole brain. Each point indicates, for a given species, the average relative cortical mass as a percentage of total brain mass (X-axis) and the average relative number of cortical neurons as a percentage of the total number of neurons in the brain (Y-axis). Data from Herculano-Houzel et al. (2006, 2007); Azevedo et al. (2009); and Sarko et al. (2009). orders, considers solely how brain size changes as a function of its number of neurons within a given order, irrespective of body size, and without any concerns regarding phylogenenetic effects within that order, or even whether evolution of the extant species has involved an expansion of brain size, a reduction, or both. In the particular case of primates, we have recently extended our analysis to another set of five primate species (Gabi et al., submitted), and found that the same cellular scaling relationships apply to the original dataset (Herculano-Houzel et al., 2007), to the second dataset, and to the combined, extended dataset. This is evidence that the cellular scaling rules considered here from a set of primate species also extend to primates as a whole, and can be used to infer the expected cellular composition of the human brain - even though small variations may occur across species that might, indeed, be due to phylogenetic interdependencies.

In the order Rodentia, we find that the brain increases in size faster than it gains neurons, with a decrease in neuronal densities which, in the presence of constant non-neuronal cell densities, implies that average neuronal size increases rapidly as neurons become more numerous (Herculano-Houzel et al., 2006). The increase in numbers of neurons in the cerebral cortex, cerebellum and remaining areas is concurrent with an even greater increase in numbers of non-neurons, yielding a maximal glia/neuron ratio that increases with brain size (Herculano-Houzel et al., 2006). These findings corroborated previous studies describing neuronal density decreasing and the glia-to-neuron ratio increasing with increasing brain size across mammalian taxa (Tower and Elliot, 1952; Shariff, 1953; Friede, 1954; Tower, 1954; Hawking and Olszewski, 1957; Haug, 1987; Reichenbach, 1989; Stolzenburg et al., 1989).

In contrast to rodent brains, which scale hypermetrically in size with their numbers of neurons, primate brain size increases approximately isometrically as a function of neuron number, with no systematic change in neuronal density or in the non-neuronal/ neuronal ratio with increasing brain size (Herculano-Houzel et al., 2007). Across insectivore species, on the other hand, the cerebellum increases linearly in size as a function of its number of neurons (as in primates), while the cerebral cortex increases in size hypermetrically as it gains neurons (as in rodents; Sarko et al., 2009). In view of the similar non-neuronal cell densities across species, hypermetric scaling of brain structure mass as a function of its number of neurons implies a concurrent increase in the average neuronal size (which, in the method's definition, includes not only the cell soma but also the entire dendritic and axonal arborizations as well as synapses; Herculano-Houzel et al., 2006). As a consequence of these different cellular scaling rules, shown in Table 1, a 10-fold increase in the number of neurons in a rodent brain results in a 35 -fold larger brain; in contrast, a similar 10-fold increase in the number of neurons in a primate brain results in an increase in brain size of only 11-fold.

\section{NOT ALL BRAINS ARE CREATED EOUAL: COGNITIVE ABILITIES AND NUMBERS OF NEURONS}

The different cellular scaling rules that apply to rodent, primate and insectivore brains show very clearly that brain size cannot be used indiscriminately as a proxy for numbers of neurons in the brain, or even in a brain structure, across orders. By maintaining the average neuronal size (including all arborizations) invariant 


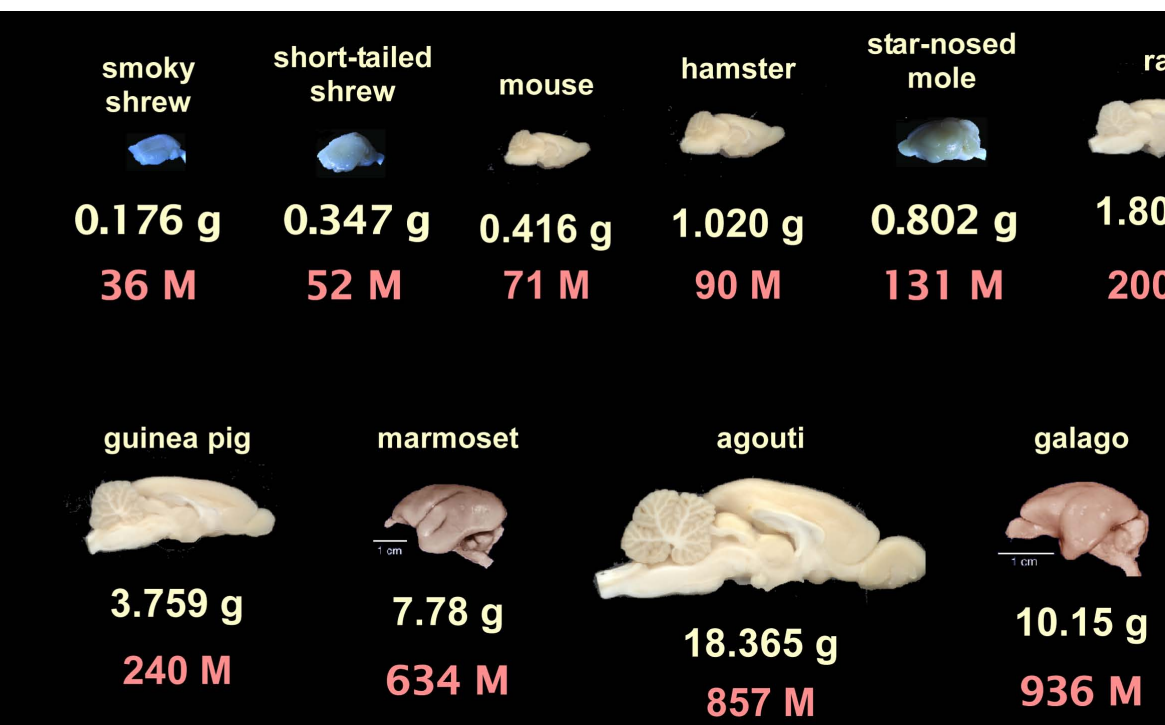

eastern mole

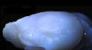

$0.999 \mathrm{~g}$

204 M

capybara

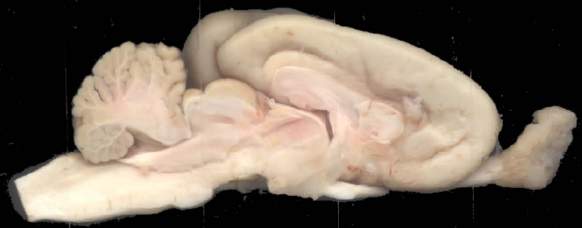

$76.036 \mathrm{~g}$

$1600 \mathrm{M}$ squirrel monkey

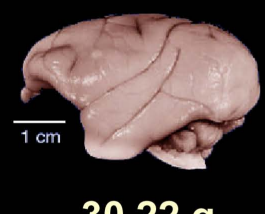

$30.22 \mathrm{~g}$

$3246 \mathrm{M}$ capuchin monkey

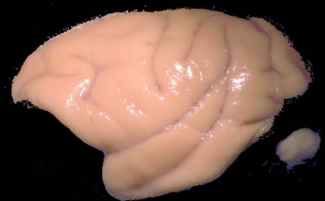

$53.21 \mathrm{~g}$ 3690 M

human

owl monkey

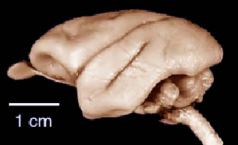

$15.73 \mathrm{~g}$

$1468 \mathrm{M}$

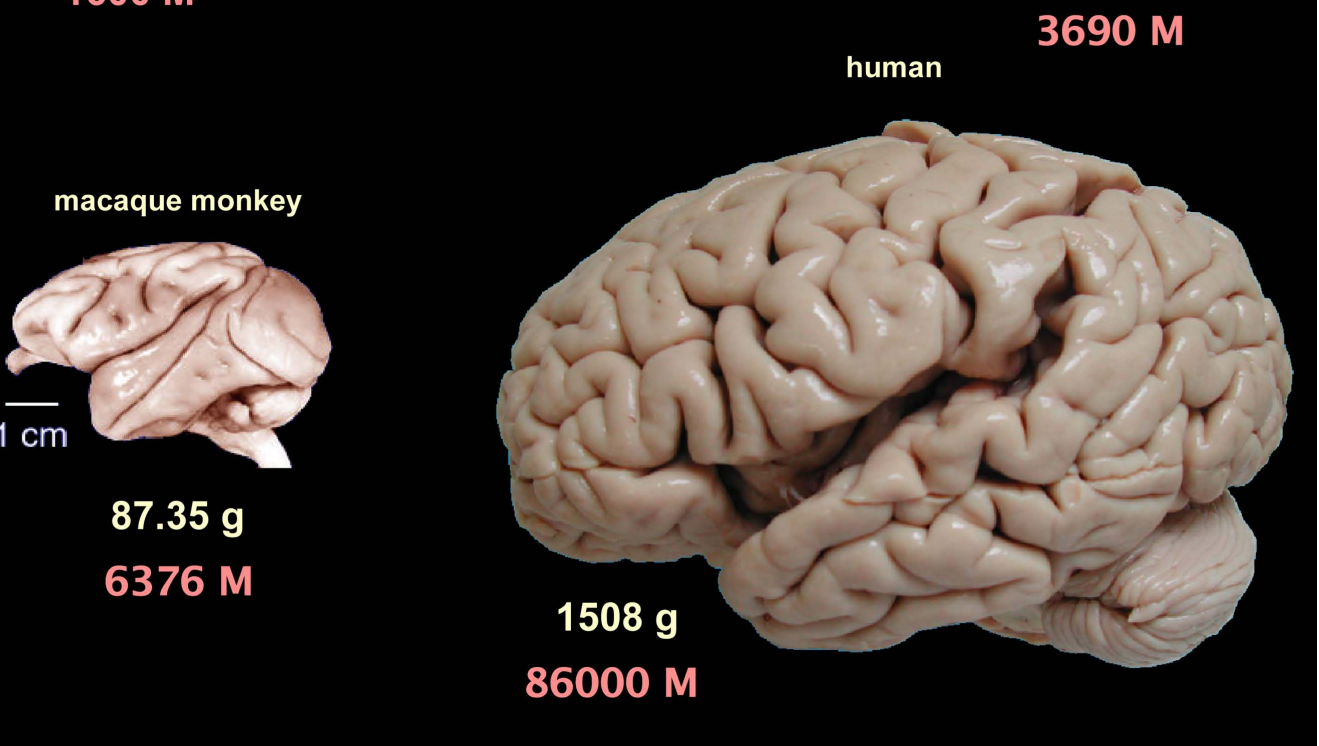

FIGURE 3 | Brain mass and total number of neurons for the mammalian species examined so far with the isotropic fractionator. Brains are arranged from left to right, top to bottom, in order of increasing number of neurons according to average species values from Herculano-Houzel et al., 2006 (rodents), Herculano-Houzel et al., 2007 (non-human primates), Sarko et al., 2009 (insectivores) and Azevedo et al., 2009 (human brain). Rodent brains face right, primate brains face left, insectivore brains can be identified in the figure by their bluish hue (due to illumination conditions). All images shown to the same scale. Primate images, except for the capuchin monkey and human brain, from the University of Wisconsin and Michigan State Comparative Mammalian Brain Collections (www.brainmuseum.org). Insectivore images kindly provided by Diana Sarko, and human brain image by Roberto Lent. Rodent images from the author. Notice that some rodent brains, such as the agouti and the capybara, contain fewer neurons than primate brains that are smaller than them. 
as brain size changes, primate brains scale in size in a much more space-saving, economical manner compared to the inflationary growth that occurs in rodents, in which larger numbers of neurons are accompanied by larger neurons.

The cognitive consequences of this difference, which allows primate brains to enjoy the benefits of a large increase in numbers of neurons without the otherwise associated cost of a much larger increase in overall brain volume, can be glimpsed by returning to the comparison between rodents and primates of similar brain size. Now that absolute numbers of neurons can be compared across the similar-sized brains of agoutis and owl monkeys, and of capybaras and capuchin monkeys (Figure 4), the expected correlation between cognitive ability and numbers of neurons is actually found to hold: with 1468 million neurons, owl monkeys have almost twice as many neurons in the brain as agoutis (which hold 857 million), and about four times more neurons in the cerebral cortex than the agouti (442 million versus 113 million). Likewise, the capuchin monkey brain has more than twice the number of neurons of the

Table 1 | Power law exponents that apply to the scaling of brain mass, or structure mass, as a function of the number of neurons they contain in rodents, insectivores and primates.

\begin{tabular}{llll}
\hline & Rodents & Insectivores & Primates \\
\hline Brain mass $\times$ neurons & $\mathrm{M}_{\mathrm{BR}} \sim \mathrm{N}_{\mathrm{BR}}^{1.550}$ & $\mathrm{M}_{\mathrm{BR}} \sim \mathrm{N}_{\mathrm{BR}}^{1.016}$ & $\mathrm{M}_{\mathrm{BR}} \sim \mathrm{N}_{\mathrm{BR}}{ }^{1.056}$ \\
Cortical mass $\times$ neurons & $\mathrm{M}_{\mathrm{CX}} \sim \mathrm{N}_{\mathrm{CX}}^{1.744}$ & $\mathrm{M}_{\mathrm{CX}} \sim \mathrm{N}_{\mathrm{CX}}^{1.520}$ & $\mathrm{M}_{\mathrm{CX}} \sim \mathrm{N}_{\mathrm{CX}}^{1.077}$ \\
Cerebellar mass $\times$ neurons & $\mathrm{M}_{\mathrm{CB}} \sim \mathrm{N}_{\mathrm{CB}}^{1.372}$ & $\mathrm{M}_{\mathrm{CB}} \sim \mathrm{N}_{\mathrm{CB}}^{1.028}$ & $\mathrm{M}_{\mathrm{CB}} \sim \mathrm{N}_{\mathrm{CB}}^{0.990}$ \\
Remaining areas & $\mathrm{M}_{\mathrm{RA}} \sim \mathrm{N}_{\mathrm{RA}}{ }^{1.153}$ & $\mathrm{M}_{\mathrm{RA}} \sim \mathrm{N}_{\mathrm{RA}}{ }^{0.926}$ & $\mathrm{M}_{\mathrm{RA}} \sim \mathrm{N}_{\mathrm{RA}}{ }^{1.013}$ \\
mass $\times$ neurons & & &
\end{tabular}

Data are from Herculano-Houzel et al. (2006), Sarko et al. (2009) and HerculanoHouzel et al. (2009). Scaling laws for primate brains do not include human values. larger-brained capybara (3.7 billion against 1.6 billion), and also about four times more neurons in the cerebral cortex ( 1.1 billion against 0.3 ).

The significance of the difference in scaling rules for building brains with large numbers of neurons becomes even more obvious if one considers the expected number of neurons for a generic rodent brain of human-sized proportions, weighing $1.5 \mathrm{~kg}$ : such a brain would have only 12 billion neurons, and a much larger number of 46 billion non-neuronal cells. This number of neurons is smaller than the number of neurons estimated to exist in the human cerebral cortex alone (Pakkenberg and Gundersen, 1997; Pelvig et al., 2008), and about seven times smaller than the number of neurons predicted for a $1.5-\mathrm{kg}$ brain built with the scaling rules that apply to primates (see below).

\section{THE CELLULAR COMPOSITION OF THE HUMAN BRAIN}

The determination of the cellular scaling rules that apply to primate brains (Herculano-Houzel et al., 2007) enabled us to predict the cellular composition of the human brain. According to these rules, a generic primate brain of $1.5 \mathrm{~kg}$ should have 93 billion neurons, and 112 billion non-neuronal cells: glial cells, thus, should constitute at most half of all brain cells. This generic primate brain should have a cerebral cortex of about $1.4 \mathrm{~kg}$, containing 25 billion neurons, and a cerebellum weighing $120 \mathrm{~g}$, with 70 billion neurons (Table 2).

Establishing whether the human brain indeed conforms to the scaling rules that apply to other primates, however, required determining its cellular composition using the same method. This was accomplished by Azevedo et al. (2009), who found that the adult male human brain, at an average of $1.5 \mathrm{~kg}$, has 86 billion neurons and 85 billion non-neuronal cells - numbers that deviate from the expected by 7 and $24 \%$ only. The human cerebral cortex, with an average $1233 \mathrm{~g}$ and 16 billion neurons, is slightly

\section{Rodents Primates}
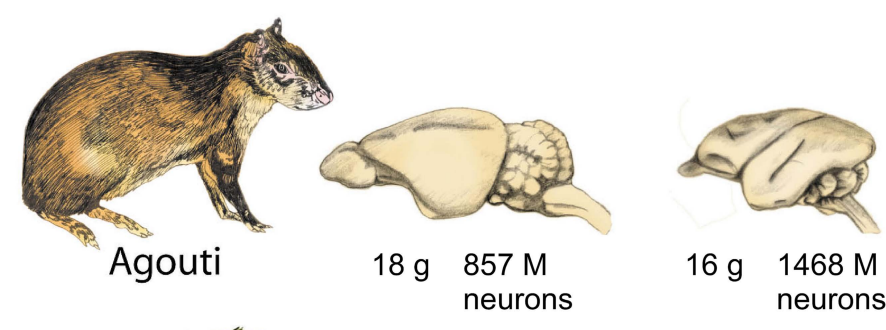

$16 \mathrm{~g} 1468 \mathrm{M}$ neurons
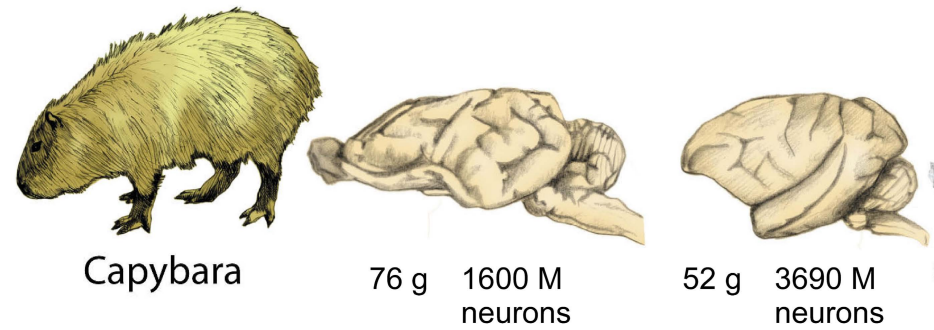

$52 \mathrm{~g} \quad 3690 \mathrm{M}$ neurons

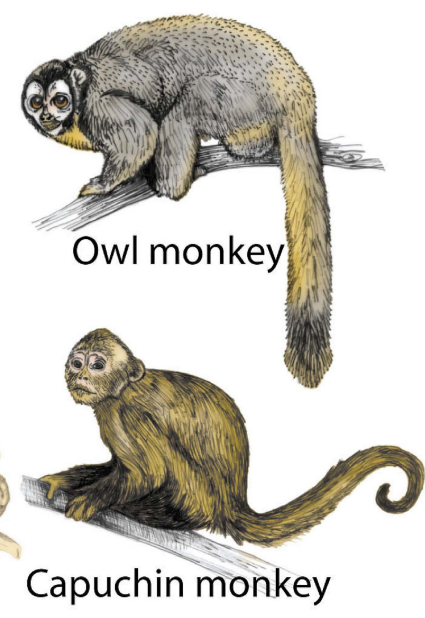

FIGURE 4 | Brain size is not a reliable indicator of number of neurons across orders. Because of the different cellular scaling rules that apply to rodent and primate brains, primates always concentrate larger numbers of neurons in the brain than rodents of a similar, or even larger, brain size. Data from Herculano-Houzel et al. (2006, 2007). Illustration by Lorena Kaz. 
Table 2 | Expected values for a generic rodent and primate brains of $\mathbf{1 . 5} \mathbf{~ k g}$, and values observed for the human brain (Azevedo et al., 2009).

\begin{tabular}{|c|c|c|c|}
\hline & Generic rodent brain & Generic primate brain & Human brain \\
\hline Brain mass & $1500 \mathrm{~g}$ & $1500 \mathrm{~g}$ & $1508 \mathrm{~g}$ \\
\hline Total number of neurons in brain & 12 billion & 93 billion & 86 billion \\
\hline Total number of non-neurons in brain & 46 billion & 112 billion & 85 billion \\
\hline Mass, cerebral cortex & $1154 \mathrm{~g}$ & $1412 \mathrm{~g}$ & $1233 \mathrm{~g}$ \\
\hline Neurons, cerebral cortex & 2 billion & 25 billion & 16 billion \\
\hline Relative size of the cerebral cortex & $77 \%$ of brain mass & $94 \%$ of brain mass & $82 \%$ of brain mass \\
\hline Relative number of neurons in cerebral cortex & $17 \%$ of brain neurons & $27 \%$ of brain neurons & $19 \%$ of brain neurons \\
\hline Mass, cerebellum & $133 \mathrm{~g}$ & $121 \mathrm{~g}$ & $154 \mathrm{~g}$ \\
\hline Neurons, cerebellum & 10 billion & 61 billion & 69 billion \\
\hline Relative size of the cerebellum & $9 \%$ of brain mass & $8 \%$ of brain mass & $10 \%$ of brain mass \\
\hline
\end{tabular}

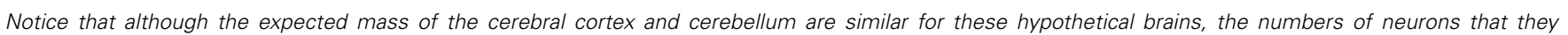

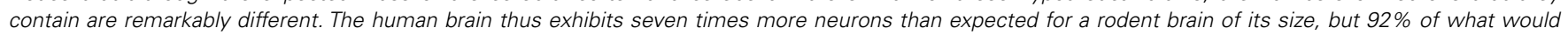

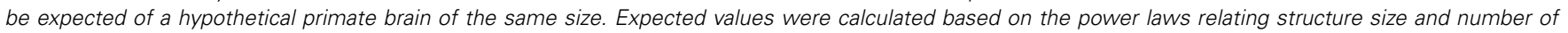
neurons (irrespective of body size) that apply to average species values for rodents (Herculano-Houzel et al., 2006) and primate brains (Herculano-Houzel et al., 2007), excluding the olfactory bulb.

below expectations for a primate brain of $1.5 \mathrm{~kg}$, while the human cerebellum, at $154 \mathrm{~g}$ and 69 billion neurons, matches or even slightly exceeds the expected (Table 2).

Although not observed in the comparatively small rodent species analyzed, the enlargement of the cerebral cortex is not, in principle, an exclusive feature of the human brain: a similar expansion of the mass of the cerebral cortex, relative to the whole brain, is predicted by both the rodent and primate cellular scaling rules, irrespective of the number of neurons contained in the cortex (Table 2). Remarkably, the human cerebral cortex, which represents $82 \%$ of brain mass, holds only $19 \%$ of all neurons in the human brain - a fraction that is similar to the fraction that we observed in several other primates, rodents, and even insectivores (Figure 1). The relatively large human cerebral cortex, therefore, is not different from the cerebral cortex of other animals in its relative number of neurons.

It should be noted that the unchanging proportional number of neurons in the cerebral cortex relative to the whole brain does not contradict an expansion in volume, function and number of neurons of the cerebral cortex in evolution: the absolute number of neurons in the rodent and primate cerebral cortex does increase much faster in larger brains compared to the number of neurons in the combined brainstem, diencephalon and basal ganglia, and is accompanied by a similarly fast increase in the number of neurons in the cerebellum (Figure 5).

Because of the diverging power laws that relate brain size and number of neurons across rodents and primates, the latter can hold more neurons in the same brain volume, with larger neuronal densities than found in rodents. Since neuronal density does not scale with brain size in primates, but decreases with increasing brain size in rodents, the larger the brain size, the larger is the difference in number of neurons across similar-sized rodent and primate brains.

\section{PREDICTIONS FOR GREAT APES}

The finding that the same cellular scaling rules apply to humans and non-anthropoid primate brains alike, irrespective of body size, indicates that the brains of the great apes, which diverged from the hominin lineage before humans, should also conform to the same cellular scaling rules. An examination of the cellular composition of the cerebellum of orangutans and one gorilla shows that the sizes of the cerebellum and cerebral cortex predicted for these species from the number of cells in the cerebellum match their actual sizes, which suggests that the brain of these animals indeed is built according to the same scaling rules that apply to humans and other primates (Herculano-Houzel and Kaas, in preparation). In view of the discrepant relationship between body and brain size in humans, great apes, and non-anthropoid primates, these findings suggest that the rules that apply to scaling primate brains are much more conserved than those that apply to scaling the body. This raises the possibility that brain mass and body mass across species are only correlated, rather than brain mass being determined by body mass, as presumed in studies that focus on the variation of residuals after regression onto body size. Supportive evidence comes from the dissociation between brain and body growth in development, in which the former actually precedes the latter (reviewed in Deacon, 1997), and from our observation that body mass seems more free to vary across species than brain mass as a function of its number of neurons. In this view, it will be interesting to consider the alternative hypothesis that body size is not a determining variable for brain size in comparative studies of brain neuroanatomy, and particularly not an (independent) parameter for assessing quantitative aspects of the human brain.

\section{DO WE HAVE THE MOST NEURONS? PREDICTIONS FOR OTHER LARGE- BRAINED MAMMALS}

The different cellular scaling rules that apply to rodents and primates strongly indicate that it is not valid to use brain size as a proxy for number of neurons across humans, whales, elephants and other large-brained species belonging to different mammalian orders. One consequence of this realization is that sheer size alone, or in relation to body size, is not an adequate parameter to qualify, or disqualify, the human brain as "special".

A comparison of expected numbers can nevertheless be very illuminating. For instance, given the cellular scaling rules that we have observed for rodents (Herculano-Houzel et al., 2006), a hypothetical 

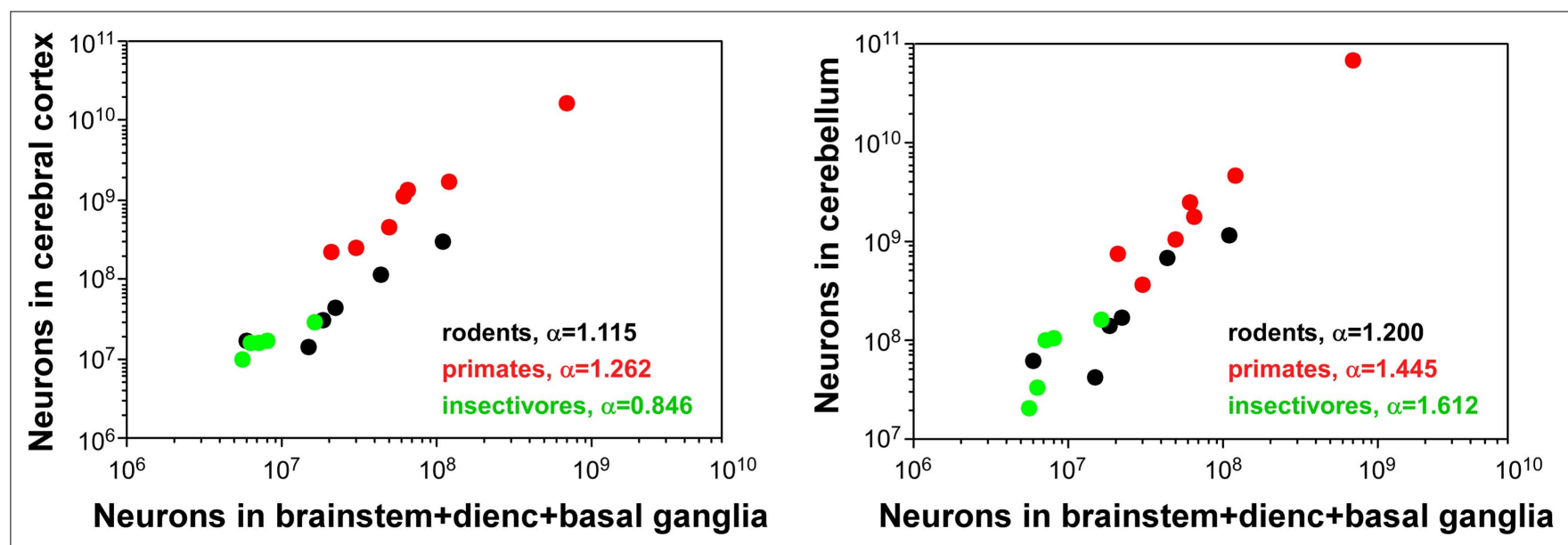

FIGURE 5 | Numbers of neurons increase faster in the cerebral cortex and cerebellum than in the remaining brain areas (the combined brainstem, diencephalon and basal ganglia). Data points indicate average values for individual species of rodents (Herculano-Houzel et al., 2006), primates (Herculano-Houzel et al., 2007), including humans (Azevedo et al., 2009), and insectivores (Sarko et al., 2009).

rodent brain with 86 billion neurons, like the human brain, would be predicted to weigh overwhelming $35 \mathrm{~kg}$ - a value that is way beyond the largest known brain mass of $9 \mathrm{~kg}$ for the blue whale, and probably physiologically unattainable. As mentioned above, a generic rodent brain of human-sized proportions, weighing $1.5 \mathrm{~kg}$, would have only 12 billion neurons: in this sense, therefore, being a primate endows us with seven times more neurons than would be expected if we were rodents. Notice that this remarkable difference does not rely on assumptions about how brain size or cellular composition relate to body size in the species.

A burning question is now whether cetaceans and elephants, endowed with much larger brains than humans, also have much larger numbers of neurons than humans. According to one estimate, the false killer whale and the African elephant would have about 11 billion neurons in the cerebral cortex, despite their large size - and fewer neurons than the 11.5 billion estimated by the same method for the human cerebral cortex, though only marginally so (Roth and Dicke, 2005). These estimates, however, were obtained by simply multiplying cerebral cortical volume and the neuronal densities determined for a few cortical areas, which probably do not reflect average neuronal density in the entire cortex.

Although direct measurements of cellular composition are not yet available from whole elephant and whale brains, it is illuminating to consider how their cellular compositions would differ depending on whether predicted from the scaling rules that apply to rodent or to primate brains. As shown in Table 3, the difference in numbers of neurons predicted to compose the brains of the false killer whale and of the African elephant is 10-fold depending on the scaling rules employed. Speculatively, the estimate of neuronal density in the gray matter of the cerebral cortex of the whale and the elephant at a low figure of about 7000 neurons $/ \mathrm{mm}^{3}$ (Tower, 1954) suggests that these brains conform to scaling rules that are much closer to those that apply to rodents than to the primate scaling rules. It may turn out, therefore, these very large brains are composed of remarkably fewer neurons than the human brain, despite their size, thanks to the distinct, economical scaling rules that apply to primates in general (and not to humans in particular).

\section{THE HUMAN BRAIN IS A LINEARLY SCALED-UP PRIMATE BRAIN IN ITS NUMBER OF NEURONS. WHAT NOW? COGNITIVE ABILITIES, BRAIN SIZE AND NUMBER OF NEURONS}

To conclude that the human brain is a linearly scaled-up primate brain, with just the expected number of neurons for a primate brain of its size, is not to state that it is unremarkable in its capabilities. However, as studies on the cognitive abilities of non-human primates and other large-brained animals progress, it becomes increasingly likely that humans do not have truly unique cognitive abilities, and hence must differ from these animals not qualitatively, but rather in the combination and extent of abilities such as theory of mind, imitation and social cognition (Marino et al., 2009). Quantitative changes in the neuronal composition of the brain could therefore be a main driving force that, through the exponential combination of processing units, and therefore of computational abilities, leads to events that may look like "jumps" in the evolution of brains and intelligence (Roth and Dicke, 2005). Such quantitative changes are likely to be warranted by increases in the absolute (rather than relative) numbers of neurons in relevant cortical areas and, coordinately, in the cerebellar circuits that interact with them (Ramnani, 2006). Moreover, viewing the human brain as a linearly scaled-up primate brain in its cellular composition does not diminish the role that particular neuroanatomical arrangements, such as changes in the relative size of functional cortical areas (for instance, Semendeferi et al., 2001; Rilling and Seligman, 2002), in the volume of prefrontal white matter (Schoenemann et al., 2005) or in the size of specific portions of the cerebellum (Ramnani, 2006) may play in human cognition. Rather, such arrangements should contribute to brain function in combination with the large number of neurons in the human brain. Our analysis of numbers of neurons has so far been restricted to large brain divisions, such as the entire cerebral cortex and the ensemble of brainstem, diencephalon and basal ganglia, but an analysis of the cellular scaling of separate functional cortical areas and the related subcortical structures is underway. Such data should allow us to address important issues such as mosaic evolution through concerted changes in the functionally related 
Table 3 | Predicted cellular composition of whale and elephant brains if they scaled according to rodent or primate cellular scaling rules.

Predicted from rodent rules

Predicted from primate rules

\section{FALSE KILLER WHALE, 3650 G}

Neurons, whole brain

Mass, cerebral cortex

Neurons, cerebral cortex

Neuronal density, cortex

Mass, cerebellum

Neurons, cerebellum

Neuronal density, cerebellum

\section{AFRICAN ELEPHANT, 4200 G}

Neurons, whole brain

Mass, cerebral cortex

Neurons, cerebral cortex

Neuronal density, cortex

Mass, cerebellum

Neurons, cerebellum

Neuronal density, cerebellum

21 billion
$3000 \mathrm{~g}$
3 billion
1000 neurons $/ \mathrm{mg}^{*}$
$304 \mathrm{~g}$
19 billion
63,500 neurons/mg*

23 billion

$3488 \mathrm{~g}$

3 billion

960 neurons/mg *

$347 \mathrm{~g}$

21 billion

61,200 neurons/mg*
212 billion

$3655 \mathrm{~g}$

55 billion

30-80,000 neurons/mg * *

$279 \mathrm{~g}$

140 billion

400-600,000 neurons/mg *
241 billion

$4245 \mathrm{~g}$

62 billion

30-80,000 neurons/mg***

$318 \mathrm{~g}$

159 billion

400-600,000 neurons/mg *

Neuronal densities $\left(^{*}\right)$ predicted from the rodent scaling rules apply to the whole structures, including white matter. Neuronal densities predicted from the primate scaling rules are the range observed in primate gray matter (**) (Herculano-Houzel et al., 2008), and in the primate cerebellum including white matter (***), since neuronal density does not covary with structure size in primates (Herculano-Houzel et al., 2007). Notice the difference in predicted numbers of neurons depending on the scaling rules applied. Given the low neuronal densities observed in the whale and elephant gray matter, of about 7000 neurons/mm ${ }^{3}$ of gray matter, it is reasonable to speculate that the scaling rules that apply to whale and elephant brains are closer to the rules that apply to rodent brains than to the rules that apply to primate brains. Notice also that the actual size of the elephant cerebellum, at about $1 \mathrm{~kg}$ (Hakeem et al., 2005), is much larger than the predicted here.

components of distributed systems, and the presumed increase in relative number of neurons in systems that increase in importance (Barton and Harvey, 2000; Barton, 2006).

If cognitive abilities among non-human primates scale with absolute brain size (Deaner et al., 2007) and brain size scales linearly across primates with its number of neurons (Herculano-Houzel et al., 2007), it is tempting to infer that the cognitive abilities of a primate, and of other mammals for that matter, are directly related to the number of neurons in its brain. In this sense, it is interesting to realize that, if the same linear scaling rules are considered to apply to great apes as to other primates, then similar three-fold differences in brain size and in brain neurons alike apply to humans compared to gorillas, and to gorillas compared to baboons. This, however, is not to say that any cognitive advantages that the human brain may have over the gorilla and that the gorilla may have over the baboon are equally three-fold - although these differences are difficult to quantify. Since neurons interact combinatorially through the synapses they establish with one another, and further so as they interact in networks, the increase in cognitive abilities afforded by increasing the number of neurons in the brain can be expected to increase exponentially with absolute number of neurons, and might even be subject to a thresholding effect once critical points of information processing are reached. In this way, the effects of a three-fold increase in numbers of neurons may be much more remarkable when comparing already large brains, such as those of humans and gorillas, than when comparing small brains, such as those of squirrel monkeys and galagos.

\section{INTRASPECIFIC VARIABILITY IN SIZE, NUMBERS AND ABILITIES}

One final caveat to keep in mind when studying scaling of numbers of brain neurons, particularly in regard to cognition, is that relationships observed across species need not apply to comparisons across individuals of the same species. Not only the extent of intraspecific variation is much smaller (on the order of $10-50 \%$ ) than interspecific variation (which spans five orders of magnitude within mammals; Tower, 1954; Stolzenburg et al., 1989), but also the mechanisms underlying interspecific and intraspecific variation are also likely to differ. Our own preliminary data suggest that, indeed, variations in brain size across rats of the same age are not correlated with variations in numbers of neurons (Morterá and HerculanoHouzel, unpublished observations). There is no justification, therefore, to extend the linear correlation between brain size and number of neurons across primates to a putative correlation across persons of different brain sizes (which might be used, inappropriately, as grounds for claims that larger-brained individuals have more neurons, and are therefore "smarter", than smaller-brained persons). In fact, although men have been reported to have more neurons in the cerebral cortex than women (Pakkenberg and Gundersen, 1997; Pelvig et al., 2008), there is no significant correlation between brain size and general cognitive ability within families (Schoenemann et al., 2000). Across these individuals, other factors such as variations in number and identity of synaptic connections within and across structures, building on a statistically normal, albeit variable, number of neurons, and depending on genetics and life experiences such as learning, are more likely to be determinant of the individual cognitive abilities (see, for instance, Mollgaard et al., 1971; Black et al., 1990; Irwin et al., 2000; Draganski et al., 2004).

\section{CONCLUDING REMARKS: OUR PLACE IN NATURE}

Novel quantitative data on the cellular composition of the human brain and its comparison to other primate brains strongly indicate that we need to rethink our notions about the place that the human brain holds in nature and evolution, and rewrite some of the basic concepts 
that are taught in textbooks. Accumulating evidence (Deacon, 1997; Roth and Dicke, 2005; Deaner et al., 2007) indicates that an alternative view of the source of variations in cognitive abilities across species merits investigation: one that disregards body and brain size and examines absolute numbers of neurons as a more relevant parameter instead. Now that these numbers can be determined in various brains and their structures, direct comparisons can be made across species and orders, with no assumptions about body-brain size relationships required. Complementarily, however, it now becomes possible to examine how numbers of neurons in the brain, rather than brain size, relate to body mass and surface as well as metabolism, parameters that have been considered relevant in comparative studies (Martin, 1981; Fox and Wilczynski, 1986; MacLarnon, 1996; Schoenemann, 2004), in order to establish what mechanisms underlie the loosely correlated scaling of body and brain.

According to this now possible neuron-centered view, rather than to the body-centered view that dominates the literature (see Gazzaniga, 2008, for a comprehensive review), the human brain has the number of neurons that is expected of a primate brain of its size; a cerebral cortex that is exactly as large as expected for a primate brain of $1.5 \mathrm{~kg}$; just as many neurons as expected in the cerebral cortex for the size of this structure; and, despite having a relatively large cerebral cortex (which, however, a rodent brain of $1.5 \mathrm{~kg}$ would also be predicted to have), this enlarged cortex holds just the same proportion of brain neurons in humans as do other primate cortices (and rodent cortices, for that matter). This final observation calls for a reappraisal of the view of brain evolution that concentrates on the expansion of the cerebral cortex, and its replacement with a more integrated view of coordinate evolution of cellular composition, neuroanatomical structure, and function of cerebral cortex and cerebellum (Whiting and Barton, 2003).

\section{REFERENCES}

Allen, N. J., and Barres, B.A. (2009). Glia more than just brain glue. Nature 457, 675-677.

Andersen, B. B., Korbo, L., and Pakkenberg, B. (1992). A quantitative study of the human cerebellum with unbiased stereological techniques. J. Comp. Neurol. 326, 549-560.

Azevedo, F. A., Carvalho, L. R., Grinberg, L. T., Farfel, J. M., Ferretti, R. E., Leite, R. E., Jacob Filho, W., Lent, R., and HerculanoHouzel, S. (2009). Equal numbers of neuronal and nonneuronal cells make the human brain an isometrically scaled-up primate brain. J. Comp. Neurol. 513, 532-541.

Barton, R. A. (2002). How did brains evolve? Nature 415, 134-135.

Barton, R. A. (2006). Primate brain evolution: integrating comparative, neurophysiological, and ethological data. Evol. Anthropol. 15, 224-236.

Barton, R. A., and Harvey, P. H. (2000). Mosaic evolution of brain structure in mammals. Nature 405, 1055-1058.

Black, J. E., Isaacs, K. R., Anderson, B. J., Alcantara, A. A., and Greenough, W T. Schuierer, G., Bogdahn, U., and

Other "facts" that deserve updating are the ubiquitous quote of 100 billion neurons (a value that lies outside of the margin of variation found so far in human brains; Azevedo et al., 2009), and, more strikingly, the widespread remark that there are $10 \times$ more glial cells than neurons in the human brain. As we have shown, glial cells in the human brain are at most $50 \%$ of all brain cells, which is an important finding since it is one more brain characteristic that we share with other primates (Azevedo et al., 2009).

Finally, if being considered the bearer of a linearly scaled-up primate brain does not sound worthy enough for the animal that considers himself the most cognitively able on Earth, one can note that there are, indeed, two advantages to the human brain when compared to others - even if it is not an outlier, nor unique in any remarkable way. First, the human brain scales as a primate brain: this economical property of scaling alone, compared to rodents, assures that the human brain has many more neurons than would fit into a rodent brain of similar size, and possibly into any other similar-sized brain. And second, our standing among primates as the proud owners of the largest living brain assures that, at least among primates, we enjoy the largest number of neurons from which to derive cognition and behavior as a whole. It will now be interesting to determine whether humans, indeed, have the largest number of neurons in the brain among mammals as a whole.

\section{ACKNOWLEDGMENTS}

Thanks to Roberto Lent and Jon Kaas for continued support and encouragement, to Bruno Mota for insightful discussions, and to the colleagues who participated in the quantification of the cellular composition of the brain of various species. Supported by CNPq-Ed. Universal and FAPERJ-Jovem Cientista do Estado do Rio de Janeiro.

matter induced by training. Nature 427, 311-312.

(190arning causessynaptogenesis whereas motor activity causes angiogenesis, in cerebellar cortex of adult rats. Proc. Natl. Acad. Sci. USA 87, 5568-5572.

Butti, C., Sherwood, C. C., Hakeem, A. Y., Allman, J. M., and Hof, P. R. (2009). Total number and volume of Von Economo neurons in the cerebral cortex of cetaceans. J. Comp. Neurol. 515, 243-259.

Clark, D. A., Mitra, P. P., and Wang, S. S. (2001). Scalable architecture in mammalian brains. Nature 411, 189-193.

Deacon, T. W. (1997). What makes the human brain different? Annu. Rev. Anthropol. 26, 337-357.

Deaner, R. O., Isler, K., Burkart, J., and van Schaik, C. (2007). Overall brain size, and not encephalization quotient, best predicts cognitive ability across non-human primates. Brain Behav. Evol. 70, 115-124.

Doetsch, F. (2003). The glial identity of neural stem cells. Nat. Neurosci. 6, 1127-1134.

Draganski, B., Gaser, C., Busch, V., May, A. (2004). Changes in grey
Finlay, B. L., and Darlington, R. B. (1995) Linked regularities in the development and evolution of mammalian brains. Science 268, 1578-1584.

Fox, J. H., and Wilczynski, W. (1986) Allometry of major CNS divisions: towards a reevaluation of somatic brain-body scaling. Brain Behav. Evol. 28, 157-169.

Friede, R. (1954). Quantitative share of the glia in development of the cortex. Acta Anat. 20, 290-296.

Gazzaniga, M. (2008). Human: The Science Behind What Makes us Unique. New York, Harper Collins.

Hakeem, A.Y., Hof, P. R., Sherwood, C. C. Switzer R. C. III, Rasmussen, L. E. L., and Allman, J. M. (2005). Brain of the African elephant (Loxodonta africana): neuroanatomy from magnetic resonance images. Anat. Rec. 287A, 1117-1127.

Hakeem, A. Y., Sherwood, C. C., Bonar, C. J., Butti, C., Hof, P. R., and Allman, J. M. (2009). Von Economo neurons in the elephant brain. Anat. Rec. 292, 242-248.
Harvey, P. H., and Pagel, M. D. (1991). Modeling physiological and anthropometric variables known to vary with body size and other confounding variables. Yearb. Phys. Anthropol. 48, 141-153.

Haug, H. (1987). Brain sizes, surfaces, and neuronal sizes of the cortex cerebri: a stereological investigation of man and his variability and a comparison with some mammals (primates, whales, marsupials, insectivores, and one elephant). Am. J. Anat. 180, 126-142.

Hawking, A., and Olszewski, J. (1957). Glia/nerve cell index for cortex of the whale. Science 126, 76-77.

Herculano-Houzel, S. (2002). Do you know your brain? A survey on public neuroscience literacy at the closing of the decade of the brain. Neuroscientist 8, 98-110.

Herculano-Houzel, S. (2007). Encephalization, neuronal excess, and neuronal index in rodents. Anat. Rec. 290, 1280-1287.

Herculano-Houzel, S., Collins, C. E., Wong, P., and Kaas, J. H. (2007). Cellular scaling rules for primate 
brains. Proc. Natl. Acad. Sci. USA 104, 3562-3567.

Herculano-Houzel, S., Collins, C. E., Wong, P., Kaas, J. H., and Lent, R. (2008). The basic nonuniformity of the cerebral cortex. Proc. Natl. Acad. Sci. USA 105, 12593-12598.

Herculano-Houzel, S., and Lent, R. (2005). Isotropic fractionator: a simple, rapid method for the quantification of total cell and neuron numbers in the brain. J. Neurosci. 25, 2518-2521.

Herculano-Houzel, S., Mota, B., and Lent, R. (2006). Cellular scaling rules for rodent brains. Proc. Natl. Acad. Sci. USA 103, 12138-12143.

Irwin, S. A., Galvez, R., and Greenough, W. T. (2000). Dendritic spine structural anomalies in fragile$\mathrm{X}$ mental retardation syndrome. Cereb. Cortex 10, 1038-1044.

Jerison, H. (1973). Evolution of the Brain and Intelligence. New York, Academic Press.

Jerison, H. (1985). Animal intelligence as encephalization. Philos. Trans. R. Soc. Lond., B, Biol. Sci. 308, 21-35.

Kandel, E. R., Schwartz, J. H., and Jessell, T. M. (2000). Principles of Neural Science, 4th Edn. New York, McGraw-Hill, pp. 19-20.

Lange, W. (1975). Cell number and cell density in the cerebellar cortex of man and some other mammals. Cell Tissue Res. 157, 115-125.

Lefebvre, L., Reader, S. M., and Sol, D. (2004). Brains, innovations and evolution in birds and primates. Brain Behav. Evol. 63, 233-246.

MacDonald, D. W. (1981). Dwindling resources and the social behavior of capybaras (Hydrochaeris hydrochaeris) (Mammalia). J. Zool. Lond. 194, 371-391.

MacLarnon, A. (1996). The scaling of gross dimensions of the spinal cord in primates and other species. J. Hum. Evol. 30, 71-87.

Marino, L. (1998). A comparison of encephalization between odontocete cetaceans and anthropoid primates. Brain Behav. Evol. 51, 230-238.

Marino, L. (2002). Convergence of complex cognitive abilities in cetaceans and primates. Brain Behav. Evol. 59, 21-32.

Marino, L., Connor, R. C., Fordyce, R. E., Herman, L. M., Hof, P. R., Lefebvre,
L., Lusseau, D., McCowan, B. Nimchinsky, E.A., Pack, A.A., Rendell, L., Reidenberg, J. S., Reiss, D., Uhen, M. D., Van der Gucht, E., and Whitehead, H. (2009). Cetaceans have complex brains for complex cognition. PLoS Biol. 5:e139. doi:10.1371/journal. pbio.0050139

Martin, R. D. (1981). Relative brain size and basal metabolic rate in terrestrial vertebrates. Nature 293, 57-60.

Mollgaard, K., Diamond, M. C. Bennett, E. L., Rosenzweig, M. R., and Lindner, B. (1971). Quantitative synaptic changes with differential experience in rat brain. Int. J. Neurosci. 2,113-127.

Nimchinsky, E. A., Glissen, E., Allman, J. M., Perl, D. P., Erwin, J. M., and Hof, P. R. (1999). A neuronal morphologic type unique to humans and great apes. Proc. Natl. Acad. Sci. USA 96, 5268-5273.

Nishiyama, A., Yang, Z., and Butt, A (2005). What's in a name? J. Anat. 207, 687-693.

Noctor, S. C., Martinez-Cerdeno, V., and Kriegstein, A.R. (2007). Contribution of intermediate progenitor cells to cortical histogenesis. Arch. Neurol. 64, 639-642.

Nunn, C., and Barton, R. A. (2000). Allometric slopes and independent contrasts: a comparative test of Kleiber's law in primates. Am. Nat. 156, 519-533.

Pakkenberg, B., and Gundersen, H. J. G. (1988). Total numbers of neurons and glial cells in human brain nuclei estimated by the disector and the fractionator. J. Microsc. 150, 1-20.

Pakkenberg, B., and Gundersen, H. J. G. (1997). Neocortical neuron number in humans: effect of sex and age.J. Comp. Neurol. 384, 312-320.

Pelvig, D. P., Pakkenberg, H., Stark, A K., and Pakkenberg, B. (2008). Neocortical glial cell numbers in human brains. Neurobiol. Aging 29, 1754-1762.

Ramnani, N. (2006). The primate corticocerebellar system: anatomy and function. Nat. Neurosci. 7, 511-522.

Reichenbach, A. (1989). Glia:neuron index: review and hypothesis to account for different values in various mammals. Glia 2, 71-77.

Rilling, J. K. (2006). Human and nonhuman primate brains: are they allometrically scaled versions of the same design? Evol. Anthropol. 15 65-77.

Rilling, J. K., and Seligman, R. A. (2002). A quantitative morphometric comparative analysis of the primate temporal lobe. J. Hum. Evol. 42, 505-533.

Roth, G., and Dicke, U. (2005). Evolution of the brain and intelligence. Trends Cogn. Sci. 9, 250-257.

Sarko, D. K., Catania, K. C., Leitch, D. B. Kaas, J. H., and Herculano-Houzel, S. (2009). Cellular scaling rules of insectivore brains. Front. Neuroanat. 3, 8 doi:10.3389/neuro.05.008.2009

Schoenemann, P. T. (2004). Brain size scaling and body composition in mammals. Brain Behav. Evol. 63 , 47-60.

Schoenemann, P.T., Budinger, T.F., Sarich, V. M., and Wang, W. S. (2000). Brain size does not predict general cognitive ability within families. Proc. Natl. Acad. Sci. USA 97, 4932-4937.

Schoenemann, P. T., Sheehan, M. J., and Glotzer, L. D. (2005). Prefrontal white matter volume is disproportionately larger in humans than in other primates. Nat. Neurosci. 8, 242-252.

Semendeferi, K., Armostrong, E. Schleicher, A., Zilles, K., and Van Hoesen, G. W. (2001). Prefrontal cortex in humans and apes: a comparative study of area 10.Am. J. Phys. Anthropol. 114, 224.

Semendeferi, K., Lu, A., Schenker, N., and Damasio, H. (2002). Humans and great apes share a large frontal cortex Nat. Neurosci. 5, 272-276.

Shariff, G.A. (1953). Cell counts in the primate cerebral cortex. J. Comp. Neurol. $98,381-400$.

Sherwood,C.C.,Stimpson,C.D., Raghanti, M. A., Wildman, D. E., Uddin, M., Grossman, L. I., Goodman, M., Redmond, J. C., Bonar, C. J., Erwin, J. M., and Hof, P. R. (2006). Evolution of increased glia-neuron ratios in the human frontal cortex. Proc. Natl. Acad. Sci. USA 103, 13606-13611.

Stolzenburg, J. U., Reichenbach, A., and Neumann, M. (1989). Size and density of glial and neuronal cells within the cerebral neocortex of various insectivorian species. Glia 2, 78-84.

Sultan, F. (2002). Analysis of mammalian brain architecture. Nature 415 133-134.
Tower, D. B. (1954). Structural and functional organization of mammalian cerebral cortex: the correlation of neurone density with brain size; cortical neurone density in the fin whale (Balaenoptera physalus L.) with a note on the cortical neurone density in the Indian elephant. J. Comp. Neurol. 101, 19-51.

Tower, D. B., and Elliot, K. A. (1952). Activity of acetylcholine system in cerebral cortex of various unanesthetized mammals. Am. J. Physiol. 168, 747-759.

Ullian, E. M., Sapperstein, S. K., Christopherson, K. S., and Barres, B. A. (2001). Control of synapse number by glia. Science 291, 657-660.

Vallender, E. J. (2008). Exploring the origins of the human brain through molecular evolution. Brain Behav. Evol. 72, 168-177.

Whiting, B. A., and Barton, R. A. (2003). The evolution of the cortico-cerebellar complex in primates: anatomical connections predict patterns of correlated evolution. J. Hum. Evol. 44, 3-10.

Williams, R. W., and Herrup, K. (1988). The control of neuron number. Аnпu. Rev. Neurosci. 11, 423-453.

Zhang, K., and Sejnowski, T. J. (2000). A universal scaling law between gray matter and white matter of cerebral cortex. Proc. Natl. Acad. Sci. USA 97, 5621-5626.

Conflict of Interest Statement: The author declares that the research was conducted in the absence of any commercial or financial relationships that could be construed as a potential conflict of interest.

Received: 15 July 2009; paper pending published: 05 August 2009; accepted: 29 September 2009; published online: 09 November 2009.

Citation: Herculano-Houzel S (2009) The human brain in numbers: a linearly scaledup primate brain. Front. Hum. Neurosci. 3:31. doi: 10.3389/neuro.09.031.2009 Copyright $\odot 2009$ Herculano-Houzel. This is an open-access article subject to an exclusive license agreement between the author and the Frontiers Research Foundation, which permits unrestricted use, distribution, and reproduction in any medium, provided the original authors and source are credited. 